\title{
Why a new journal called Biomedical Spectroscopy and Imaging?
}

The answer to the question as to why I have called this journal Biomedical Spectroscopy and Imaging is simple: there are many journals devoted to either spectroscopy or imaging but to the best of my knowledge there are none that integrates the broad area of spectroscopy and imaging. Thanks to tremendous acceleration in technology, the timing is perfect for integration of these two methodologies so that both spatial and molecular details of complex biological systems can be determined at high resolution. The two approaches complement and supplement each other in many ways and therefore it is not surprising to see a rapid growth in studies where both methods are simultaneously applied. This growth in application has been largely helped by the availability of commercial instruments that enable users to obtain both spectral and imaging data. By far the most powerful spectroscopic and imaging methods are those based on magnetic resonance. Several Nobel prizes have been awarded to scientists who have contributed in the development of magnetic resonance imaging and spectroscopy. However, in recent years there have been great advances in optical imaging methods including those based on fluorescence, Raman, terahertz, mid-infrared and near-infrared. Other developments include the use of synchrotron radiation in the acquisition of spectroscopic and imaging data. Undoubtedly, it is an exciting time to be involved in the use of spectroscopic and imaging tools. Data can be obtained at a rapid pace and then processed and analysed using sophisticated mathematical, statistical and computational methods. This is leading to remarkable progress in our understanding of diverse biomedical systems and processes including disease diagnosis and treatment planning.

After being chosen as the Editor-in-Chief of the journal, my first task was to establish an Editorial Board for the new journal. This was not an easy task but thanks to the co-operation of my friends and colleagues from the scientific community, I have established an Editorial Board with expertise in diverse areas of spectroscopy and/or imaging. I am grateful to the Editorial Board members who have all been highly supportive of the aims and goals of the journal. I am particularly thankful to Prof. Zhiwu Yu (Tsinghua University, China) for agreeing to act as the Editor for Asia, a region where the growth of spectroscopy and imaging is one of the fastest. Scientists from different regions of the world are represented on the board and the objectives are to make the journal attractive to scientists from all corners of the globe. This is evident from the first issue of the journal which consists of contributions from authors based in Asia, America, Australia and Europe. Recruitment of editors is still ongoing with a view to include editors with expertise in techniques that are currently either not-represented or underrepresented.

Biomedical Spectroscopy and Imaging (BSI) is a multidisciplinary journal devoted to the timely publication of basic and applied research that uses spectroscopic and imaging techniques in different areas of life science including biology, biochemistry, biotechnology, bionanotechnology, environmental science, 
food science, pharmaceutical science, physiology and medicine. Scientists are encouraged to submit their work for publication in the form of original articles, brief communications, rapid communications, reviews and mini-reviews.

The journal is dedicated to providing a single forum for experts in spectroscopy and imaging as applied to biomedical problems, and also for life scientists who use these powerful methods for advancing their research work. BSI aims to promote communication, understanding and synergy across the diverse disciplines that rely on spectroscopy and imaging. It also encourages the submission of articles describing development of new devices and technologies, based on spectroscopy and imaging methods, for application in diverse areas including medicine, biomedical science, biomaterials science, environmental science, pharmaceutical science, proteomics, genomics, metabolomics, microbiology, biotechnology, genetic engineering, nanotechnology, etc.

The success of this new journal will depend on many factors, the most important of which is the preparedness of the scientific community to share their knowledge and experience in the application of spectroscopy and imaging methods in unravelling the mysteries of biological systems.

Dr. Parvez I. Haris Editor-in-Chief Leicester, $U K$ 